\title{
Etude de l'écoulement tridimensionnel et instationnaire dans un rotor d'une éolienne Savonius
}

\author{
Study of the flow three-dimensional and instationary in a rotor of the
} Savonius wind turbine

\author{
Francis Raveloson ${ }^{1}$, Delphin Tomboravo ${ }^{2}$, Roger Vony ${ }^{3}$ \\ ${ }^{1}$ Laboratoire MFSEA, Université d'Antsiranana, Madagascar, ravelosonfrancis@gmail.com \\ ${ }^{2}$ Laboratoire MFSEA, Université d'Antsiranana, Madagascar, dtomboravo@gmail.com \\ ${ }^{3}$ Laboratoire MFSEA, Université d'Antsiranana, Madagascar, tsilaidrano@gmail.com
}

\begin{abstract}
RÉSUMÉ. L'objet de la présente étude est d'élaborer un code de calcul pour la résolution des équations régissant l'écoulement des fluides dans une éolienne à axe vertical. Afin de vérifier l'efficacité de notre code, nous avons fait une application sur le rotor Savonius. L'écoulement des fluides dans une roue tournante est défini par les équations de continuité, de Navier-Stokes et de l'énergie. Ces équations sont résolues numériquement par la méthode de différence finie explicite de Lax-Wendroff suivi de l'addition d'une viscosité artificielle corrigée suivant le temps. La précision spatiotemporelle de notre schéma est d'ordre deux et la stabilité du calcul numérique est assurée par la condition CFL qui impose une contrainte sur le pas de temps. Les résultats sont présentés par des courbes de pression et de Mach.

ABSTRACT. The purpose of this study is to develop a computational code for solving the equations governing the flow of fluids in a vertical axis wind turbine. In order to check the effectiveness of our code, we made an application on the Savonius rotor. The equations that define the flow of fluids in a rotating wheel are that of continuity, Navier-Stokes and energy. These equations are solved numerically by the explicit Lax-Wendroff finite difference method followed by the addition of a time-corrected artificial viscosity. The precision in the space and in the time of our scheme is second order and the stability of the numerical computation is ensured by the CFL condition which imposes a constrain on the time step. The results are presented by pressure and Mach curves.

MOTS-CLÉS. Rotor Savonius, Simulation instationnaire, aérodynamique, turbomachine.

KEYWORDS. Savonius rotor, unsteady simulation, aerodynamics, turbomachinery.
\end{abstract}

\section{Introduction}

L'énergie du vent devient la source alternative le plus utile surtout dans le domaine industriel et énergétique. De nombreux travaux de recherche ont été effectués pour valoriser la performance d'une éolienne à axe horizontal [NOU 15] et [SOS 18]. Malgré son rendement faible pour une éolienne à axe vertical [ZHA 17], [GHA 14] et [WID 12] elle devient un sujet d'actualité pour améliorer leur fonctionnement [SON 16] et [ELK 15]. Parmi les diverses études qui ont déjà traité cette question, certaines ont procédé expérimentalement à l'aide d'une soufflerie avec des instruments de mesure, en variant le nombre des pales [HAD 13] ou encore les formes [SOS 18], mais, elles demandent l'existence d'un laboratoire très puissant. D'autres études sont numériques et utilisent des logiciels de CFD [DOB 12], [MAR 17] et [WAH 13] pré-définie tels qu'Ansys-Fluent [KAN 13], [DEB 13] et [DWI 16], le "SolidWorks Flow Simulation" [WID 12] ou encore MCC+ [BUT 13] pour rentabiliser une éolienne. Toutefois, ces logiciels sont tous commerciaux et très chers ; ce qui freinent certains pays du tiers-monde de les utiliser. En plus, le logiciel Fluent est utilisé dans la plupart de cas pour la résolution numérique des équations URANS [NOU 15]. Ainsi, une approche purement tridimensionnelle à l'aide d'un logiciel libre pour déterminer la structure fine de l'écoulement travers les pales des éoliennes à axe vertical reste encore très rare. 
Face à ces problèmes, nous avons effectué dans la présente étude une simulation numérique d'un écoulement tridimensionnel et instationnaire dans une éolienne à axe vertical de type Savonius. En définitive, la simulation à l'aide d'un logiciel libre Fortran sera utilisée pour étudier la structure aérodynamique de ce rotor. Cette simulation numérique sera effectuée avec la méthode de discrétisation explicite dont le schéma adopté est celui de Lax à deux pas de temps afin d'augmenter la précision des résultats. En plus, la simulation en CFD sert à calculer les efforts aérodynamiques ainsi que le couple mécanique recueilli au bout d'arbre de ce capteur.

\section{Modélisation mathématique du problème}

\subsection{Présentation d'un modèle physique}

La géométrie du domaine de calcul et celle d'une éolienne de Savonius sont présentées dans la figure 1. Les données géométriques que nous avons utilisés dans cette étude sont les suivantes: le recouvrement $\mathrm{e}=15 \mathrm{~cm}$, le rayon $\mathrm{R}=50 \mathrm{~cm}$ et la hauteur $\mathrm{H}=120 \mathrm{~cm}$.
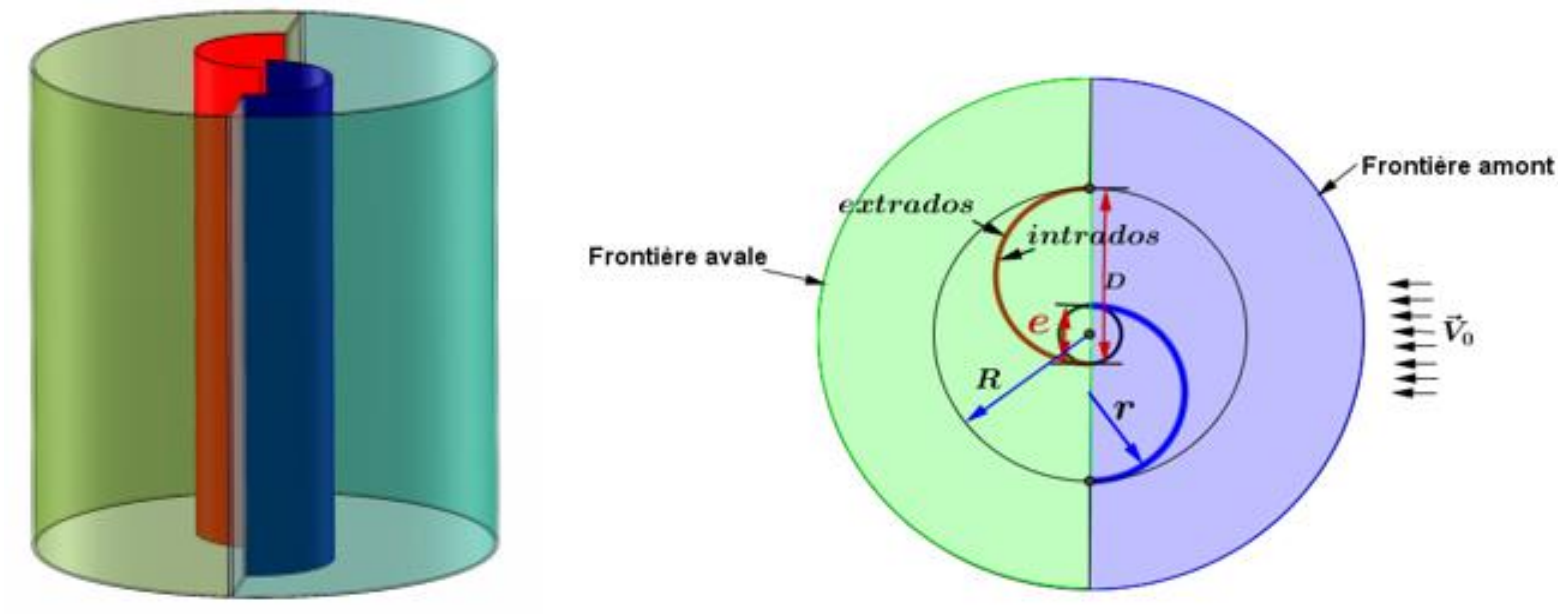

Figure 1. Description d'un rotor Savonius et du champ de calcul

Pour décrire mathématiquement le mouvement d'un fluide, celui-ci est considéré comme un milieu continu. Il obéit donc aux principes fondamentaux de la mécanique et de la thermodynamique. Trois repères sont utilisés dans cette étude :

$\checkmark$ le repère absolu formé par trois vecteurs unitaires $\vec{\imath}, \vec{\jmath}$ et $\vec{k}$;

$\checkmark$ le repère relatif orthonormé noté (E) en rotation autour d'un axe qui est défini par trois vecteurs unitaires attachés à un point $\mathrm{M}$ situé dans l'écoulement $\left(\mathrm{M}, \vec{e}_{m}, \vec{e}_{y}, \vec{e}_{n}\right)$;

$\checkmark$ et un autre repère mobile dit (H) défini par trois vecteurs unitaires $\vec{h}_{m}, \vec{h}_{n}$ et $\vec{h}_{y}$, attaché aux points du maillage (pseudo nappe de courant). 

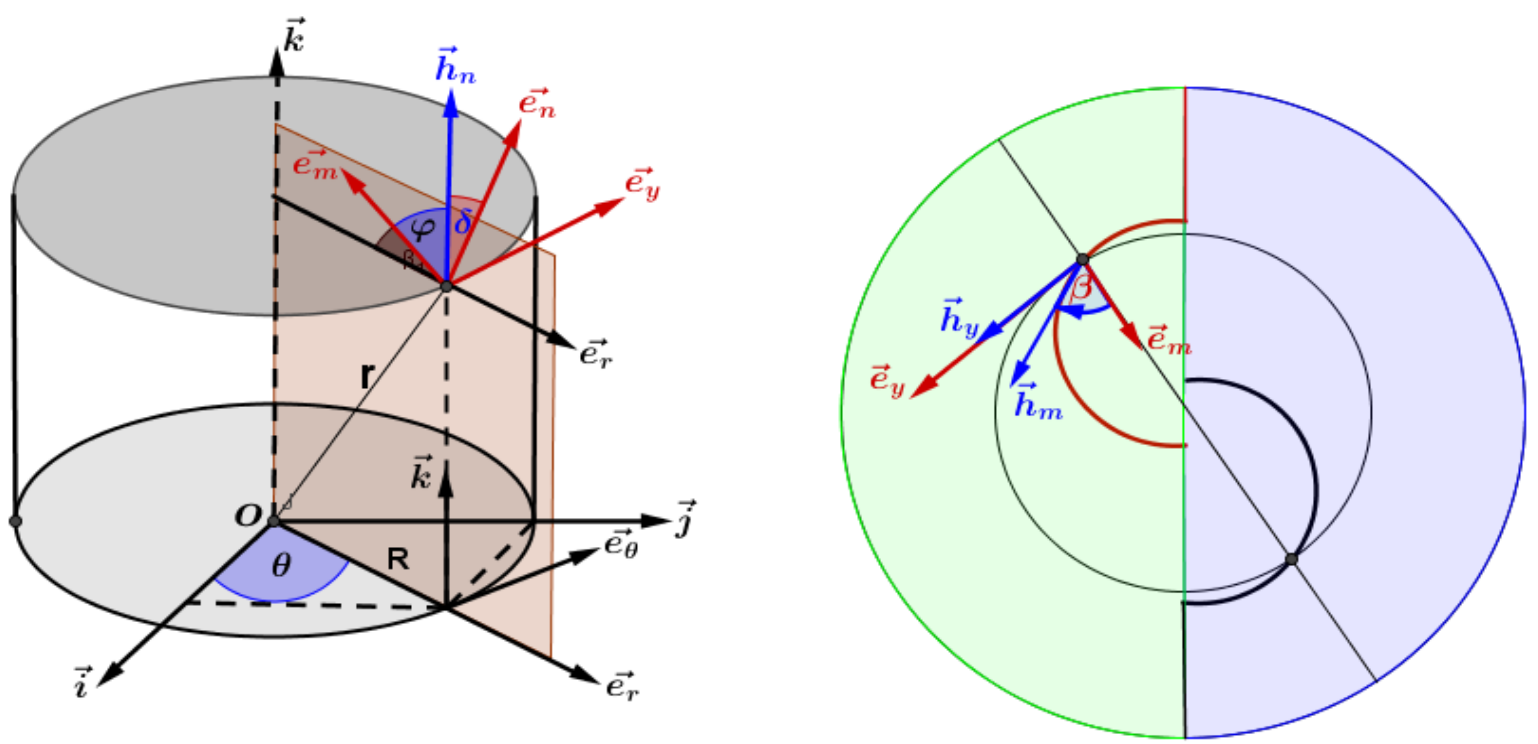

Figure 2. Présentation de trois repères utilisés

\subsection{Hypothèses simplificatrices}

Dans cette étude, pour mieux résoudre les problèmes, mais sans trop varier les phénomènes physiques qui existent, nous utilisons des hypothèses simplificatrices suivantes :

$\checkmark$ le fluide est un gaz parfait ;

$\checkmark$ le fluide est non pesant;

$\checkmark$ la conductivité thermique est négligeable.

Compte tenu des hypothèses simplificatrices ci-dessus, les équations de mouvement dans le repère mobile (H) sont écrites :

$\checkmark$ équation de continuité :

$\frac{\partial \rho \prime}{\partial t}=W_{m} \frac{\partial \rho \prime}{\partial m}+\varepsilon \frac{\partial \rho \prime}{\partial y}+W_{n} \frac{\partial \rho \prime}{\partial n}+\frac{\partial W_{m}}{\partial m}+\frac{\partial W_{y}}{\partial m}-\tan \beta \frac{\partial W_{m}}{\partial y}-\tan \delta \frac{\partial W_{n}}{\partial y}+\frac{\partial W_{n}}{\partial n}-W_{m}\left(\frac{\sin \varphi}{r}+\frac{\partial \varphi}{\partial n}\right)+$
$W_{n}\left(\frac{\cos \varphi}{r}+\frac{\partial \varphi}{\partial m}\right)$

$\checkmark$ équation de conservation de la quantité de mouvement (équation d'Euler)

$$
\begin{aligned}
& \frac{\partial W_{m}}{\partial t}=W_{m} \frac{\partial W_{m}}{\partial m}+\varepsilon \frac{\partial W_{m}}{\partial y}+W_{n} \frac{\partial W_{m}}{\partial n}+R_{g}\left[\frac{\partial T}{\partial m}-\tan \beta \frac{\partial T}{\partial y}\right]+\frac{a^{2}}{\gamma}\left[\frac{\partial \rho^{\prime}}{\partial m}-\tan \delta \frac{\partial \rho^{\prime}}{\partial y}\right]+\frac{W_{y}{ }^{2}}{r} \sin \varphi+ \\
& W_{n}\left(W_{n} \frac{\partial \varphi}{\partial n}+W_{m} \frac{\partial \varphi}{\partial m}\right)
\end{aligned}
$$

$$
\frac{\partial W_{y}}{\partial t}=W_{m} \frac{\partial W_{y}}{\partial m}+\varepsilon \frac{\partial W_{y}}{\partial y}+W_{n} \frac{\partial W_{y}}{\partial n}+R_{g} \frac{\partial T}{\partial m}+\frac{a^{2}}{\gamma} \frac{\partial \rho^{\prime}}{\partial m}+\left(W_{y}+2 U\right)\left(W_{n} \frac{\cos \varphi}{r}+W_{m} \frac{\sin \varphi}{r}\right)
$$

$$
\frac{\partial W_{n}}{\partial t}=W_{m} \frac{\partial W_{n}}{\partial m}+\varepsilon \frac{\partial W_{n}}{\partial y}+W_{n} \frac{\partial W_{n}}{\partial n}+R_{g}\left[\frac{\partial T}{\partial n}-\tan \beta \frac{\partial T}{\partial y}\right]+\frac{a^{2}}{\gamma}\left[\frac{\partial \rho \prime}{\partial n}-\tan \delta \frac{\partial \rho \prime}{\partial y}\right]+\frac{W_{y}^{2}}{r} \cos \varphi-
$$

$W_{m}\left(W_{n} \frac{\partial \varphi}{\partial n}+W_{m} \frac{\partial \varphi}{\partial m}\right)$ 
$\checkmark$ équation d'énergie

$$
\begin{aligned}
\frac{\partial T}{\partial t} & =\frac{a^{2}}{C_{p}}\left[\frac{\partial W_{m}}{\partial m}-\tan \beta \frac{\partial W_{m}}{\partial y}+\frac{\partial W_{y}}{\partial y}+\frac{\partial W_{n}}{\partial n}-\tan \delta \frac{\partial W_{n}}{\partial y}-W_{m}\left(\frac{\sin \varphi}{r}+\frac{\partial \varphi}{\partial n}\right)+W_{n}\left(\frac{\cos \varphi}{r}+\frac{\partial \varphi}{\partial m}\right)\right]+ \\
W_{m} \frac{\partial T}{\partial m} & +\varepsilon \frac{\partial T}{\partial y}+W_{n} \frac{\partial T}{\partial n}
\end{aligned}
$$

\section{Résolution numérique}

La grande majorité des méthodes présentées dans la littérature pour la résolution des équations d'Euler, sont des méthodes explicites dont les plus connues sont celles de Lax-Wendroff et de Mac Cormack. Elles présentent un grand intérêt pour la résolution des problèmes instationnaires du fait que ces schémas sont précis au second ordre par rapport au temps et l'espace. La méthode de résolution présentée dans cet article est celle de Lax-Wendroff qui a été largement utilisée dans le calcul des écoulements dans les turbomachines.

\subsection{Présentation des maillages}
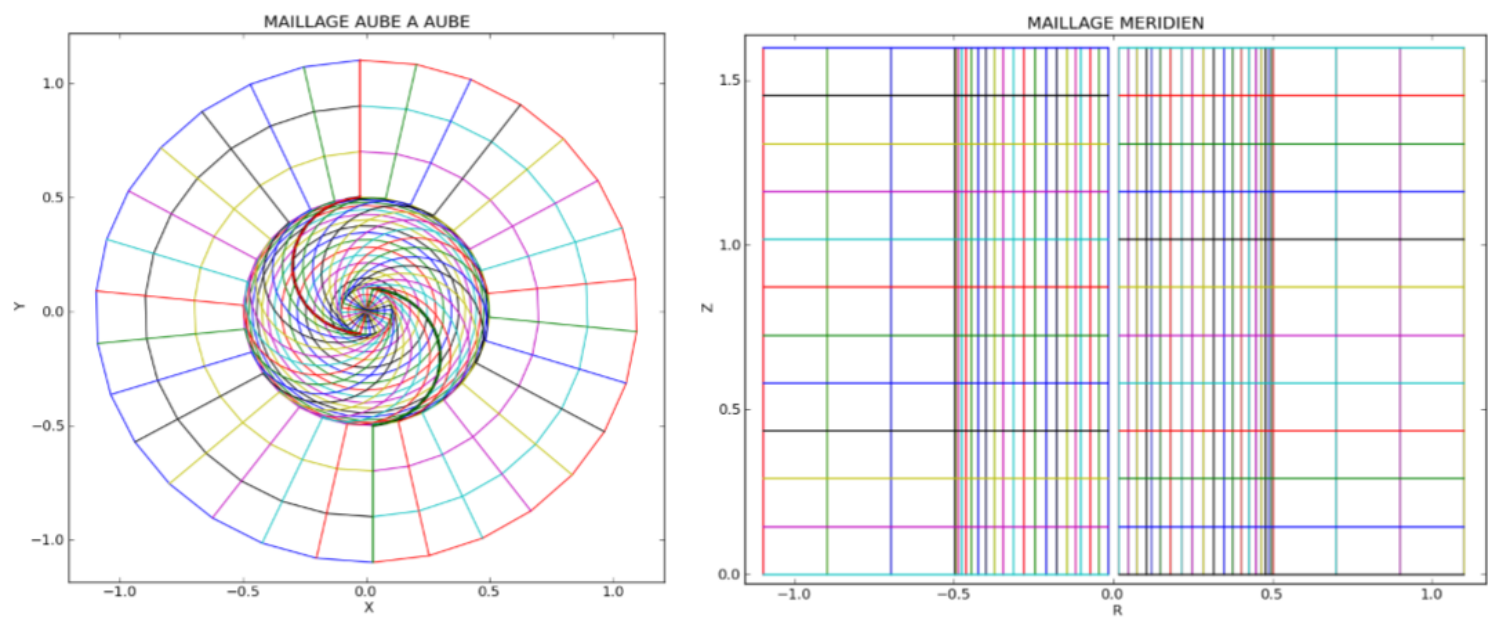

Figure 3. Maillages dans les plans aube à aube et méridien
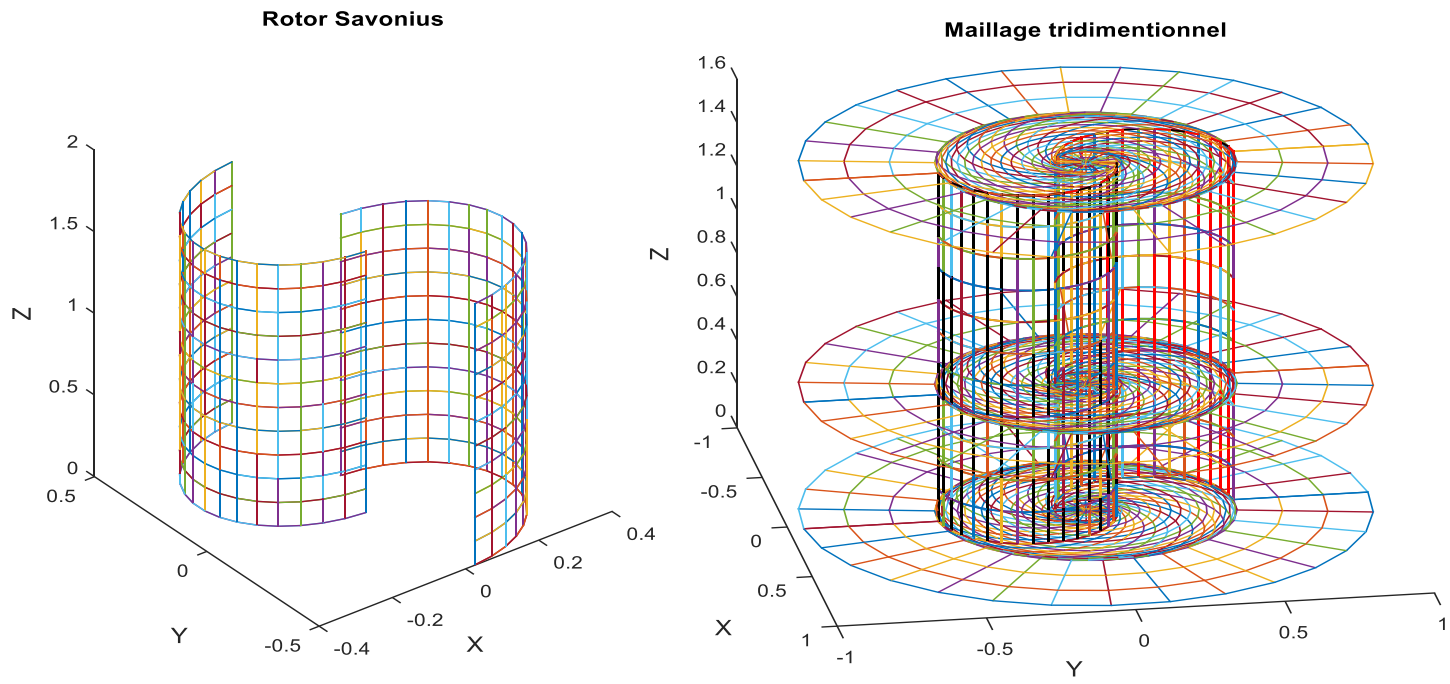

Figure 4. Maillages sur les pales et en pied, mi-hauteur et en tête des pales 
Dans le domaine de la simulation numérique de la mécanique des fluides régie par les équations de Navier-Stokes, on utilise fréquemment des maillages orthogonaux associés à des méthodes de type « différences finies » [PRU 18]. Telle est le cas de présent travail qui génère un maillage orthogonal et tridimensionnel. Ainsi, chaque paroi des pales ou des aubes correspond à une nappe de courant. Les pas de maillage sont plus lâches en dehors du canal inter aubes et plus fins au voisinage du "bord d'attaque" et du "bord de fuite". Pour ne pas surcharger la figure 4, nous avons présenté seulement un maillage en 3D au niveau du pied, à mi-hauteur, en tête et celui d'un modèle de la figure 1. Le maillage dans le plan méridien est formé par des génératrices et des droites qui leur sont perpendiculaires. Dans le plan aube à aube, il est formé par les arcs des cercles concentriques dont le centre coïncide avec l'axe de rotation de la machine.

\subsection{Discrétisation spatiale}

Le problème consiste de passer de la structure continue à une structure discrétisée équivalente [KAY 18]. Pour la discrétisation spatiale, deux cas sont à considérer : le cas des points situés à l'intérieur du domaine et le cas des points situés sur les frontières du domaine de calcul. Le schéma centré est appliqué aux points situés à l'intérieur du domaine de calcul et il se présente comme suit :

$$
\left[\frac{\partial \vec{Z}}{\partial S}\right]_{(l)}=\frac{\nabla S}{\Delta S(\nabla S+\Delta S)} \vec{Z}^{\tau}{ }_{(l+1)}+\frac{\Delta S-\nabla S}{\Delta S \nabla S} \vec{Z}_{(l)}^{\tau}-\frac{\Delta S}{\Delta S(\nabla S+\Delta S)} \vec{Z}_{(l-1)}^{\tau}
$$

Nous avons utilisé le schéma décentré vers l'intérieur pour le cas des points qui se placent sur les frontières. Sur les frontières amont, à gauche et en pied des pales, la discrétisation spatiale s'écrit :

$$
\left[\frac{\partial \vec{Z}}{\partial S}\right]_{(l)}=\frac{\nabla S}{\Delta S(\nabla S+\Delta S)} \vec{Z}_{(l+2)}^{\tau}+\frac{\Delta S-\nabla S}{\Delta S \nabla S} \vec{Z}_{(l+1)}^{\tau}-\frac{\Delta S}{\Delta S(\nabla S+\Delta S)} \vec{Z}^{\tau}{ }_{(l)}
$$

Tandis que sur les frontières aval, à droite et en tête des pales, elle devient :

$$
\left[\frac{\partial \vec{Z}}{\partial S}\right]_{(l)}=\frac{\nabla S}{\Delta S(\nabla S+\Delta S)} \vec{Z}_{(l)}^{\tau}+\frac{\Delta S-\nabla S}{\Delta S \nabla S} \vec{Z}^{\tau}{ }_{(l-1)}-\frac{\Delta S}{\Delta S(\nabla S+\Delta S)} \vec{Z}_{(l-2)}^{\tau}
$$

Dans les équations 6,7 et $8, \vec{Z}$ remplace les variables à discrétiser, $S$ la direction spatiale, $l$ l'indice correspondant à une direction considérée, $\nabla S$ et $\Delta S$ sont respectivement les pas du maillage à gauche et à droite du point étudié sauf sur les frontières.

\subsection{Discrétisation temporelle}

Une variante du schéma de Lax à deux pas de temps a été appliquée pour la discrétisation temporelle. La première étape de ce schéma correspond à celui de Lax-Friedrichs (prédicteur) et à celui de Lax-Wendroff pour la seconde (correcteur). Un schéma à viscosité numérique corrigé est également appliqué à la troisième étape de calcul dont :

$\checkmark$ schéma de Lax-Friedrich (Prédicteur)

$$
\begin{aligned}
& \vec{Z}_{(i, j, k)}^{\tau+1}=\frac{1}{6}\left(\vec{Z}_{(i+1, j, k)}^{\tau}+\vec{Z}_{(i-1, j, k)}^{\tau}+\vec{Z}_{(i, j+1, k)}^{\tau}+\vec{Z}_{(i, j-1, k)}^{\tau}+\vec{Z}_{(i, j, k+1)}^{\tau}+\vec{Z}_{(i, j, k-1)}^{\tau}\right)+\Delta t * \\
& \left(B_{m(i, j, k)}^{\tau}\left[\frac{\partial \vec{Z}}{\partial m}\right]_{(i, j, k)}^{\tau}+B_{y(i, j, k)}^{\tau}\left[\frac{\partial \vec{Z}}{\partial y}\right]_{(i, j, k)}^{\tau}+B_{n(i, j, k)}^{\tau}\left[\frac{\partial \vec{Z}}{\partial n}\right]_{(i, j, k)}^{\tau}+C_{(i, j, k)}^{\tau}\right)
\end{aligned}
$$

Où, $\tau$ est l'indice de discrétisation temporelle et $B_{m}, B_{y}, B_{n}$ et $C$ sont des variables à discrétiser selon la direction considérée représentées sous forme de matrice. 


$$
\vec{Z}_{(i, j, k)}^{\tau+2}=\vec{Z}_{(i, j, k)}^{\tau}+\Delta t *\left(B_{m(i, j, k)}^{\tau+1}\left[\frac{\partial \vec{z}}{\partial m}\right]_{(i, j, k)}^{\tau+1}+B_{y(i, j, k)}^{\tau+1}\left[\frac{\partial \vec{z}}{\partial y}\right]_{(i, j, k)}^{\tau+1}+B_{n(i, j, k)}^{\tau+1}\left[\frac{\partial \vec{z}}{\partial n}\right]_{(i, j, k)}^{\tau+1}+C_{(i, j, k)}^{\tau+1}\right)
$$

Ce schéma de discrétisation est du type dissipatif : il comporte de façon interne des termes d'amortissement, mais pas suffisants pour faire converger le calcul. Ainsi, il est nécessaire d'ajouter une viscosité numérique corrigée qui renforce les effets dissipatifs et accélère la convergence des calculs. Cependant, cette viscosité artificielle disparaîtra à la fin du calcul et l'erreur qu'elle générera n'affectera pas la précession des résultats.

$$
\begin{aligned}
& \checkmark \text { schéma à viscosité numérique corrigé } \\
& \vec{Z}_{(i, j, k)}^{\tau+3}=\vec{Z}_{(i, j, k)}^{\tau+2}-V N *\left(\vec{Z}_{(i, j, k)}^{\tau+2}-e^{*}{ }_{(i, j, k)} \vec{Z}_{(i, j, k)}^{*}\right)
\end{aligned}
$$

Avec $e_{(i, j, k)}^{*}$ le coefficient de viscosité numérique et $\vec{Z}_{(i, j, k)}^{*}$ est la variable obtenue à l'instant intermédiaire.

\subsection{Condition de stabilité du schéma}

La condition de stabilité des schémas est dictée par la condition dite CFL (Courant Friedrich Lévy) :

$$
\Delta t<\frac{h_{(i, j, k)}}{\sqrt{3}(W+a)_{(i, j, k)}}
$$

$h_{(i, j, k)}$ étant le plus petit pas du maillage dans un domaine de calcul, $W$ est la résultante de la vitesse relative et $a$ le module de la vitesse du son.

\subsection{Conditions initiales}

A l'instant initial et à travers la section perpendiculaire à la direction principale de l'écoulement $\left(\vec{e}_{m}\right)$ :

$\checkmark$ le débit reste constant: $Q=\rho W_{m} A=Q_{1}$;

$\checkmark$ la rothalpie reste constante: $I=\frac{W^{2}}{2} C_{p} T+\frac{\omega^{2} r^{2}}{2}=C^{t e}$;

$\checkmark$ en suivant les lignes du maillage, l'entropie est supposée constante (pas d'irréversibilité):

$$
\frac{\rho}{\rho_{1}}=\left(\frac{T}{T_{1}}\right)^{\frac{1}{\gamma-1}}
$$

\subsection{Conditions aux limites}

Voici les conditions aux limites que nous avons adoptées lors de la résolution numérique.

Frontière amont :

$\checkmark$ la vitesse absolue et l'angle indiquant sa direction sont fixés ;

$\checkmark$ la température est égale à la température statique.

Frontière aval : pression atmosphérique.

Frontière latérale : condition d'égalité des variables.

Parois solides (pales) : condition de glissement ;

$\mathrm{Au}$ pied et en tête des pales : condition de non-pénétration. 


\section{Validation des résultats}

Avant de présenter nos résultats de simulation numérique, il est préférable de valider le code de calcul que nous avons conçu, soit à l'aide d'étude expérimentale ou d'étude numérique déjà validée. Dans ce cas, notre code sera approuvé par l'étude de Pauline BUTAUD et al en août 2013 [BUT 13] dont les caractéristiques de géométrie et de simulation sont détaillées ci-dessous. Il s'agit d'une éolienne Savonius classique d'un mètre de diamètre et d'un recouvrement optimal de 0,15 ; ses pales en aluminium semi-cylindriques ont une épaisseur de $3 \mathrm{~mm}$. Ils ont réalisé la simulation numérique en 2D à l'aide d'un logiciel Star-CCM + dont le pas de temps choisi est de $10^{-3} \mathrm{~s}$ et le nombre de rotations du rotor nécessaire à la bonne stabilisation du système (en dynamique) est fixé à 12 tours. C'est un fluide visqueux avec un nombre de Reynolds élevé (de l'ordre de 5.10 ${ }^{6}$ ), qui le conduit naturellement à une modélisation URANS $(\mathrm{k}-\omega)$. Ils ont réalisé une étude numérique instable avec une vitesse du vent de $7 \mathrm{~m} / \mathrm{s}$ imposée à l'entrée du domaine. Les conditions aux limites sur les côtés sont des conditions de symétrie, à la sortie la pression atmosphérique et sur les aubes condition de paroi. La distribution de pression dynamique pour la position angulaire $\theta=0^{\circ}$ sera comparée à nos études en 3D.
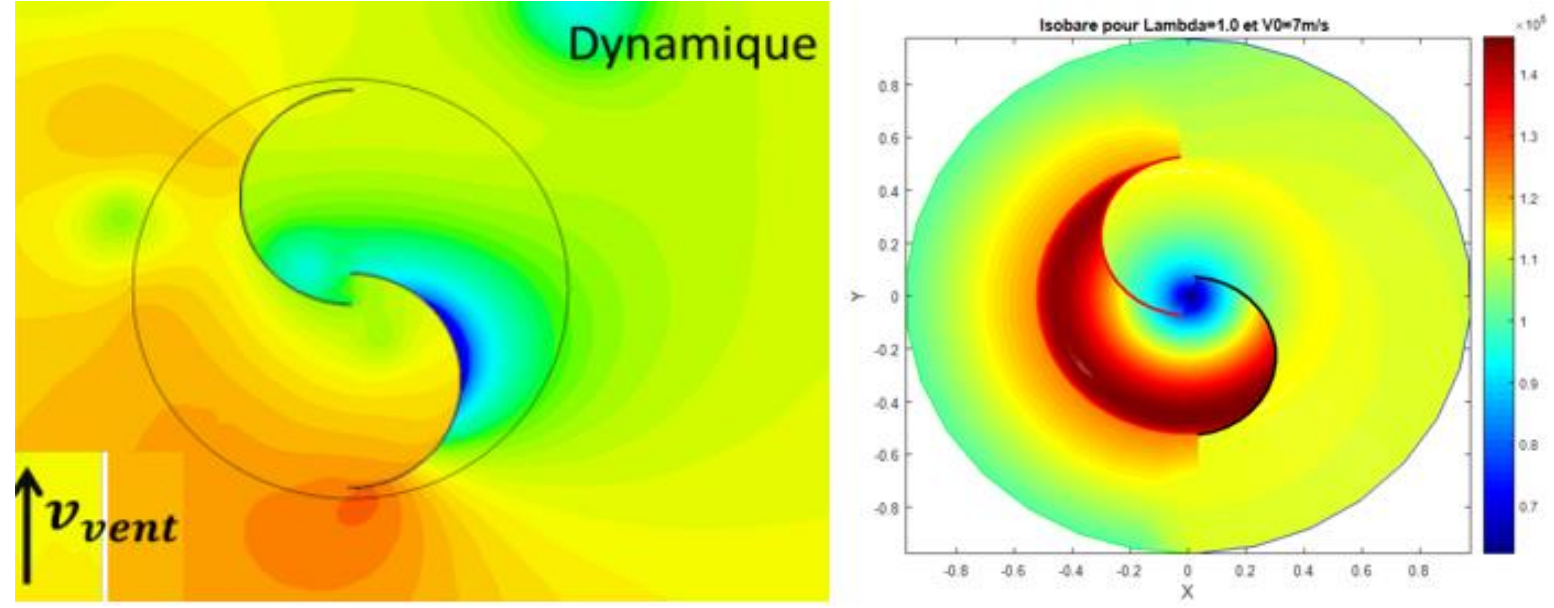

Figure 5. Distribution de pression dynamique de Pauline BUTAUD à gauche et la présente étude à droite.

\section{Résultats et discussions}

\subsection{Influence de paramètre d'avance}
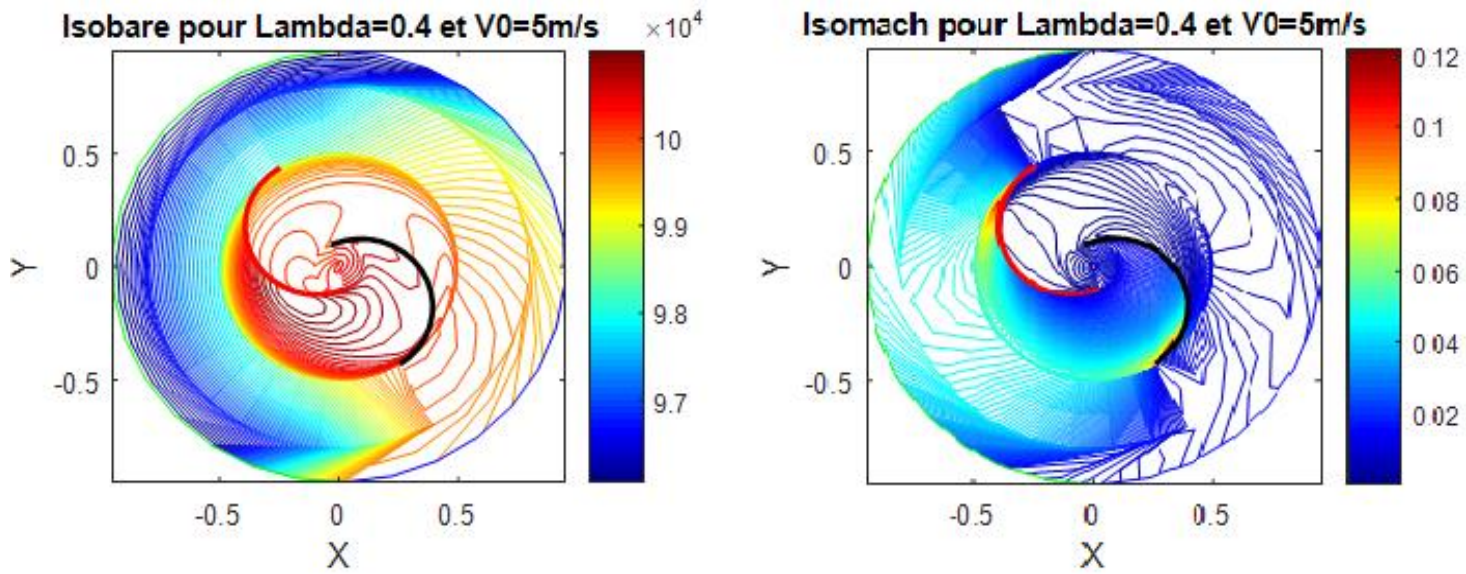

Figure 6. Courbes d'isobare et iso-Mach pour $\lambda=0,4$ 


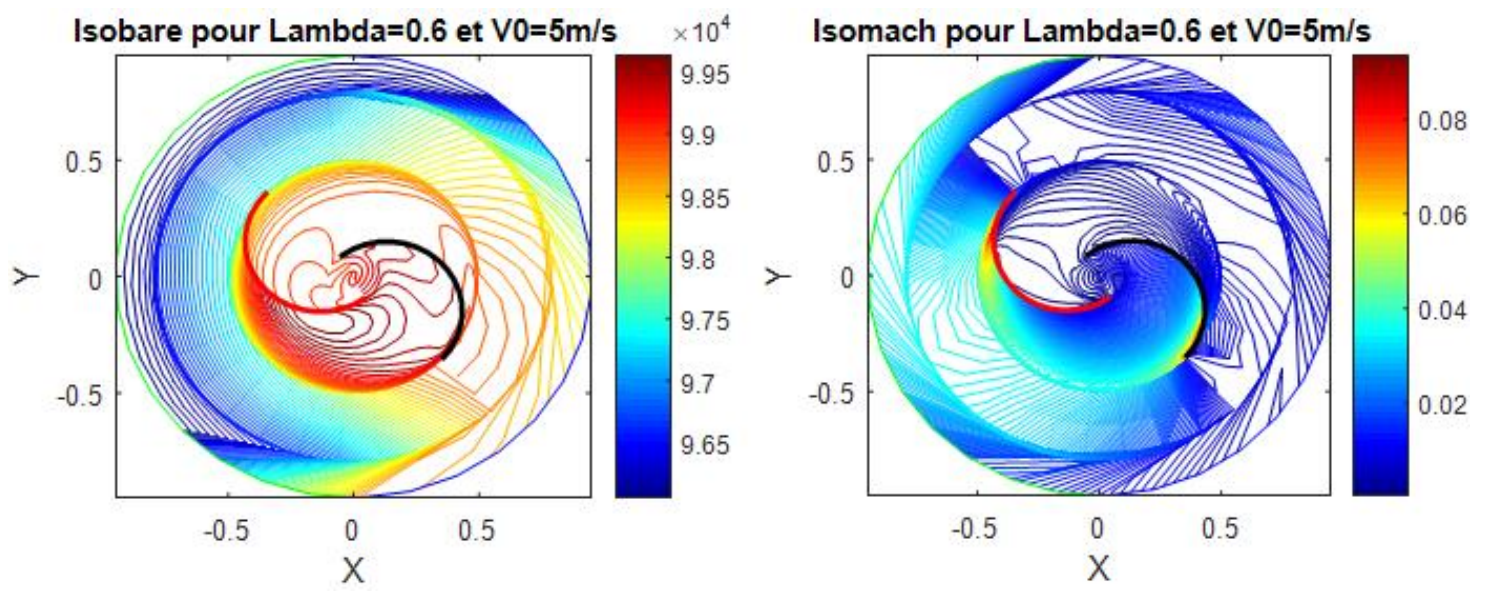

Figure 7. Courbes d'isobare et iso-Mach pour $\lambda=0,6$
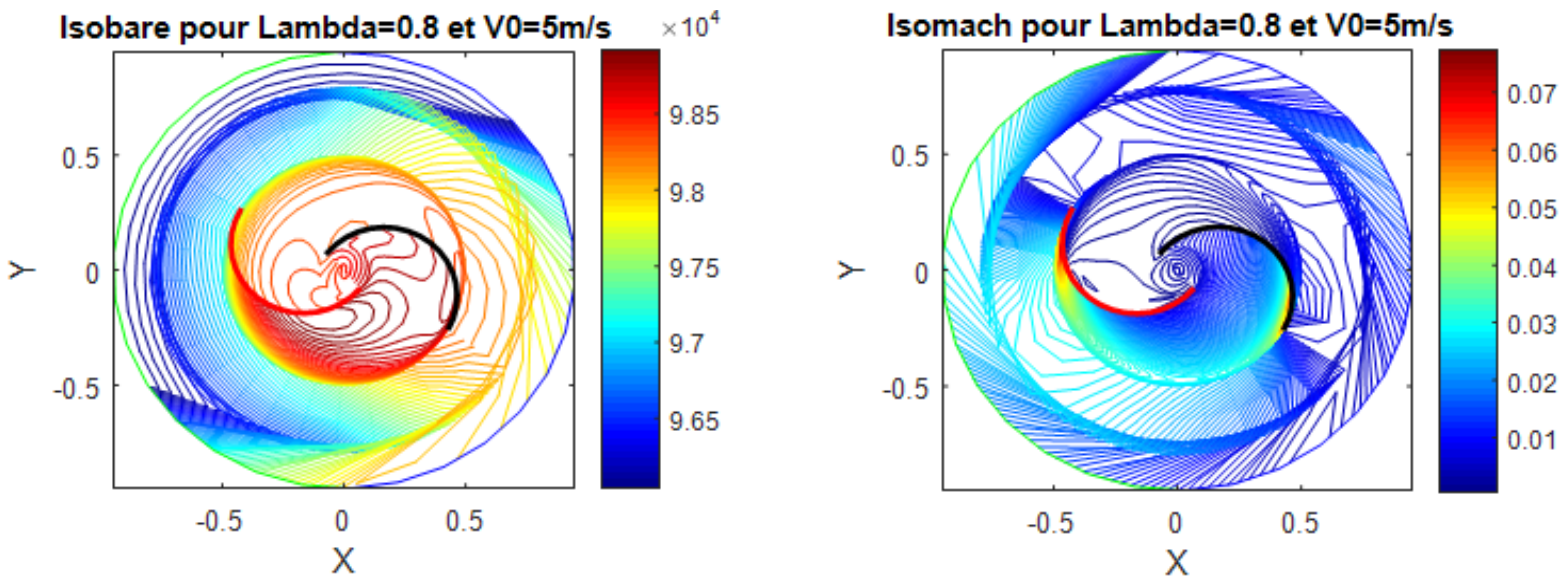

Figure 8. Courbes d'isobare et iso-Mach pour $\lambda=0,8$
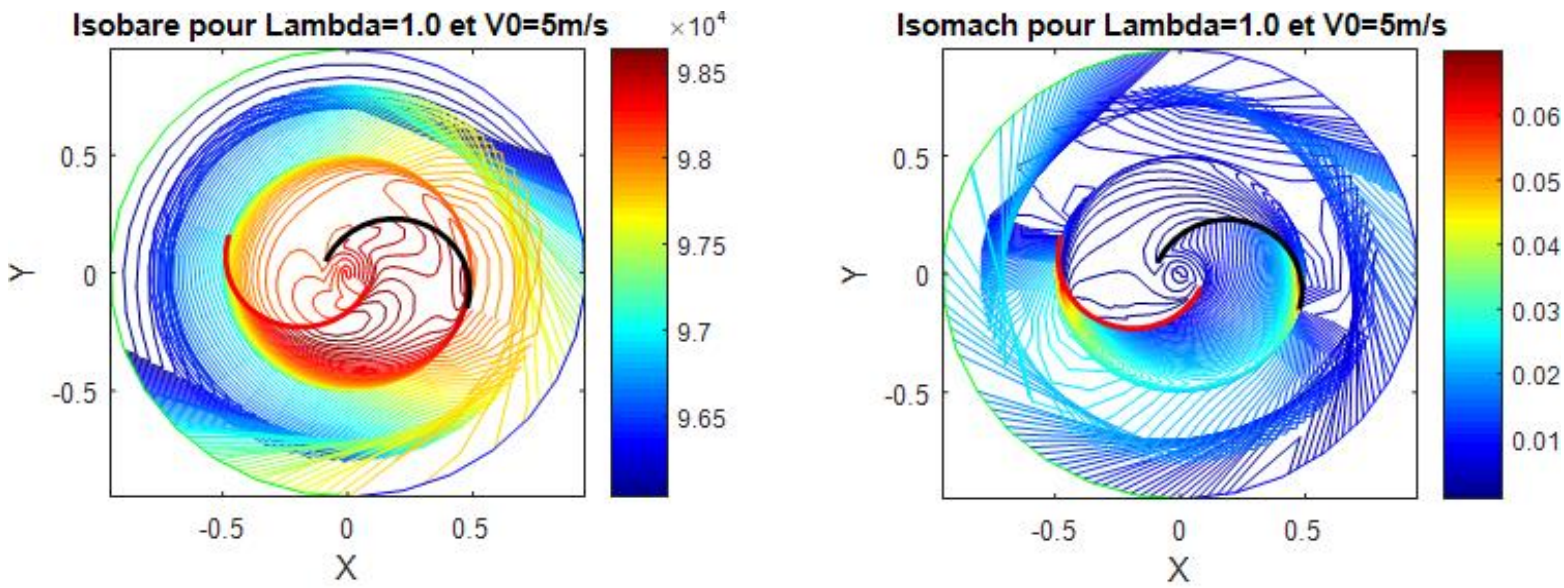

Figure 9. Courbes d'isobare et iso-Mach pour $\lambda=1,0$ 


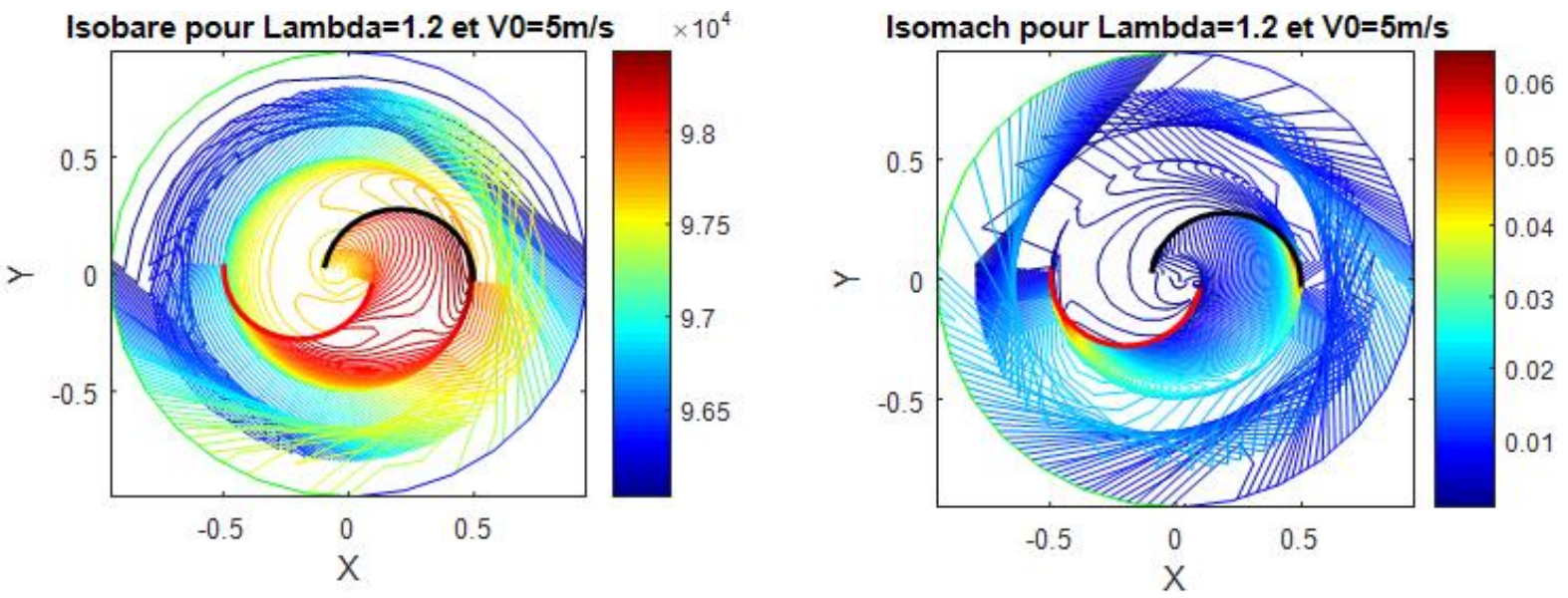

Figure 10. Courbes d'isobare et iso-Mach pour $\lambda=1,2$
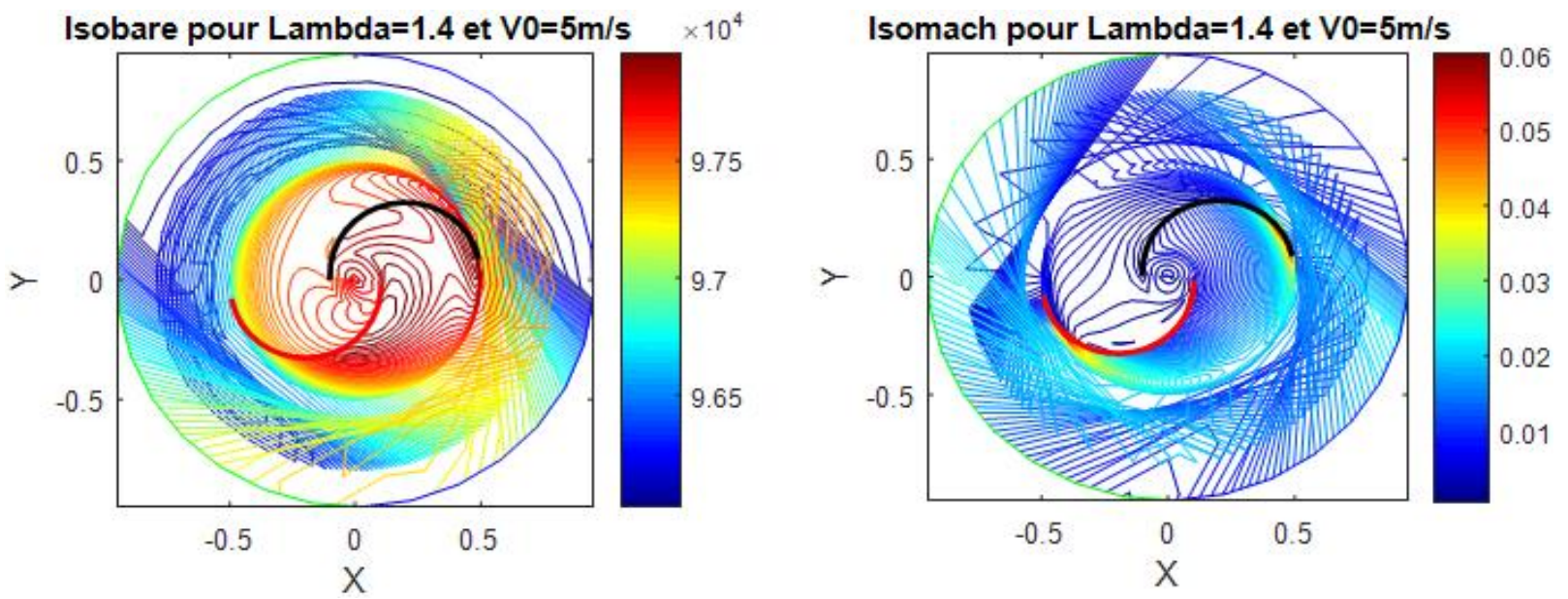

Figure 11. Courbes d'isobare et iso-Mach pour $\lambda=1,4$
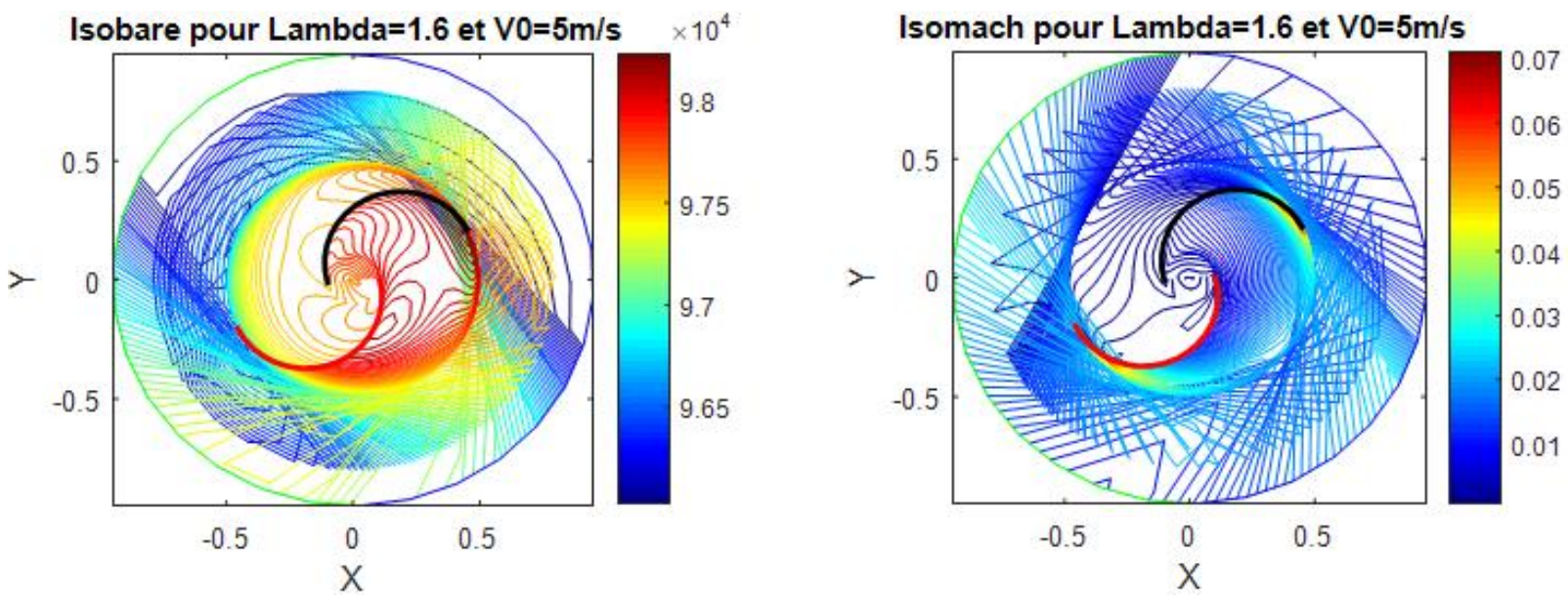

Figure 12. Courbes d'isobare et iso-Mach pour $\lambda=1,6$ 


\subsection{Influence de la position angulaire des pales}
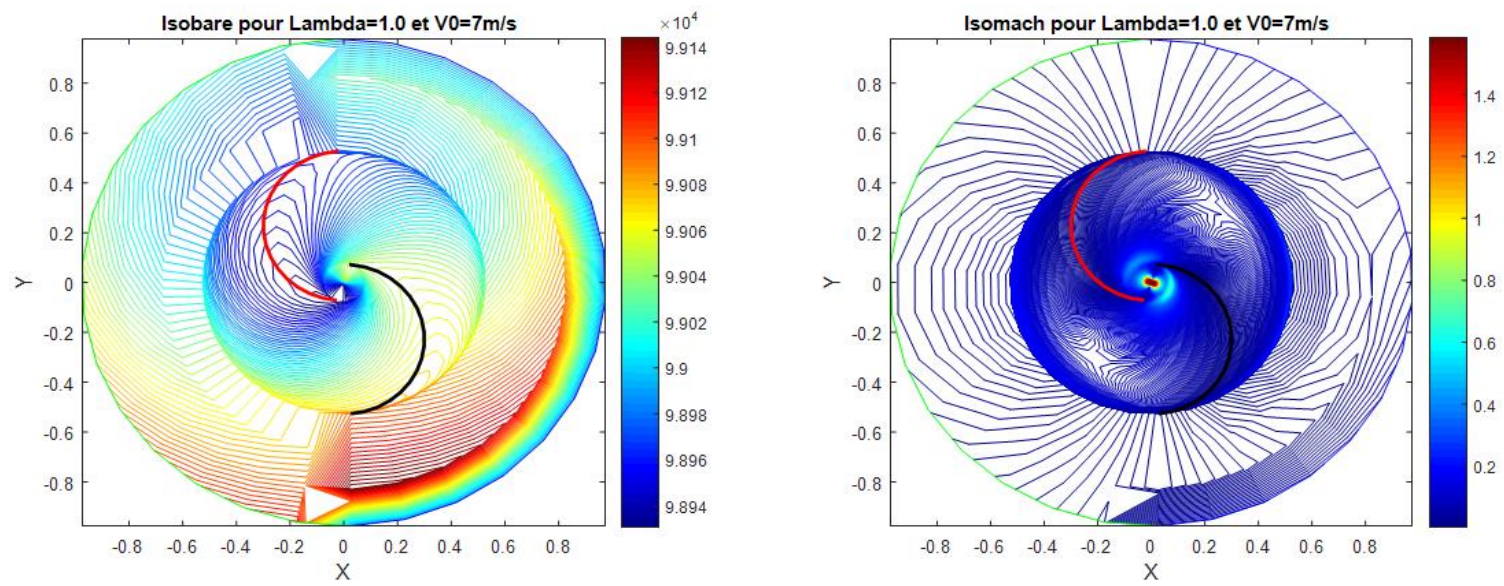

Figure 13. Courbes $d^{\prime}$ isobare et iso-Mach à $t=0$ s et $\alpha=90^{\circ}$
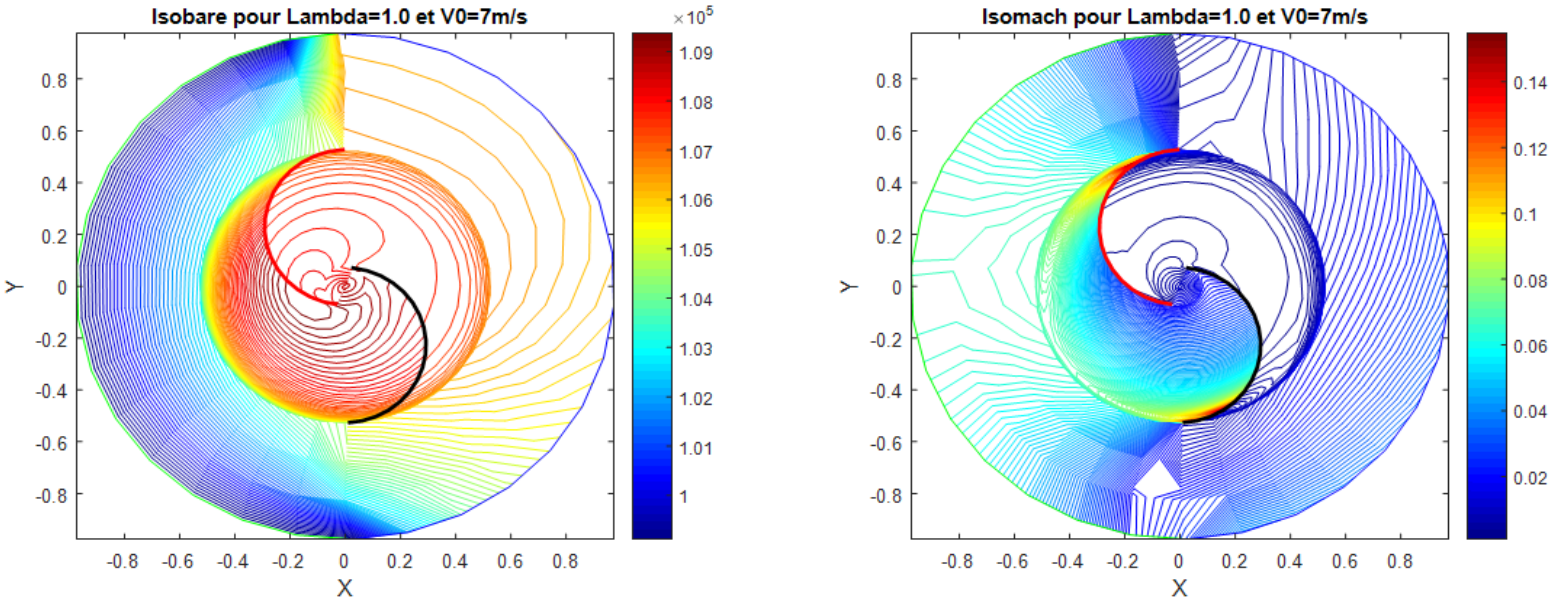

Figure 14. Courbes d'isobare à l'instant $t=0.927 s, \alpha=90^{\circ}$ après avoir effectué un tour
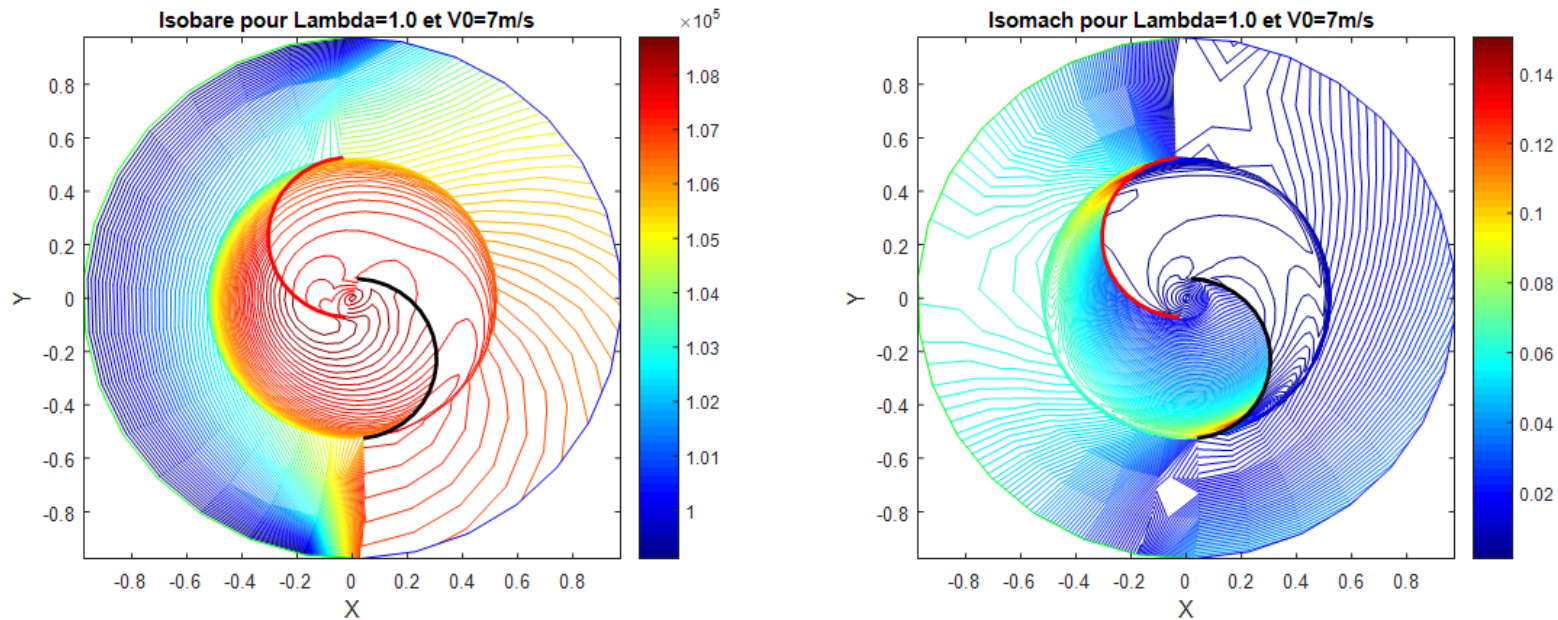

Figure 15. Courbes d'isobare à l'instant $t=5.677 \mathrm{~s}, \alpha=90^{\circ}$ après avoir effectué six tours 

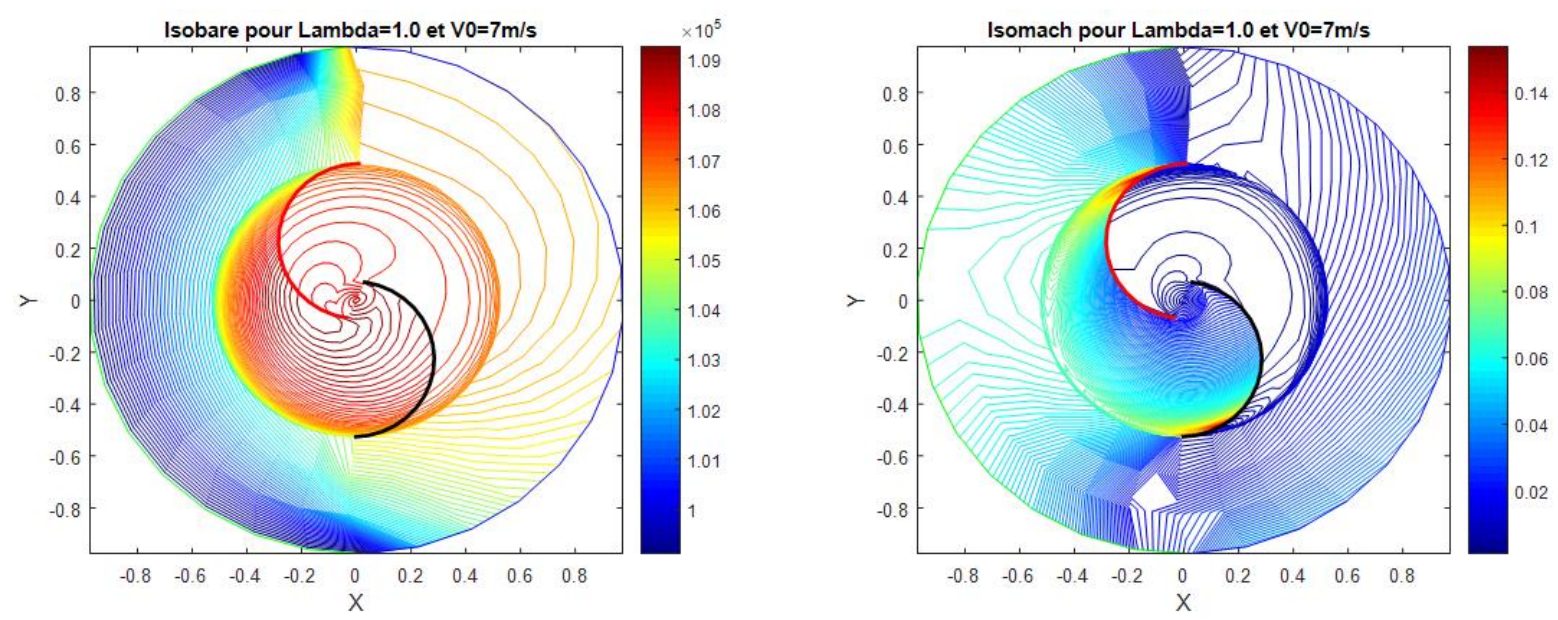

Figure 16. Courbes d'isobare à l'instant $t=11.35 \mathrm{~s}, \alpha=90^{\circ}$ après avoir effectué douze tours
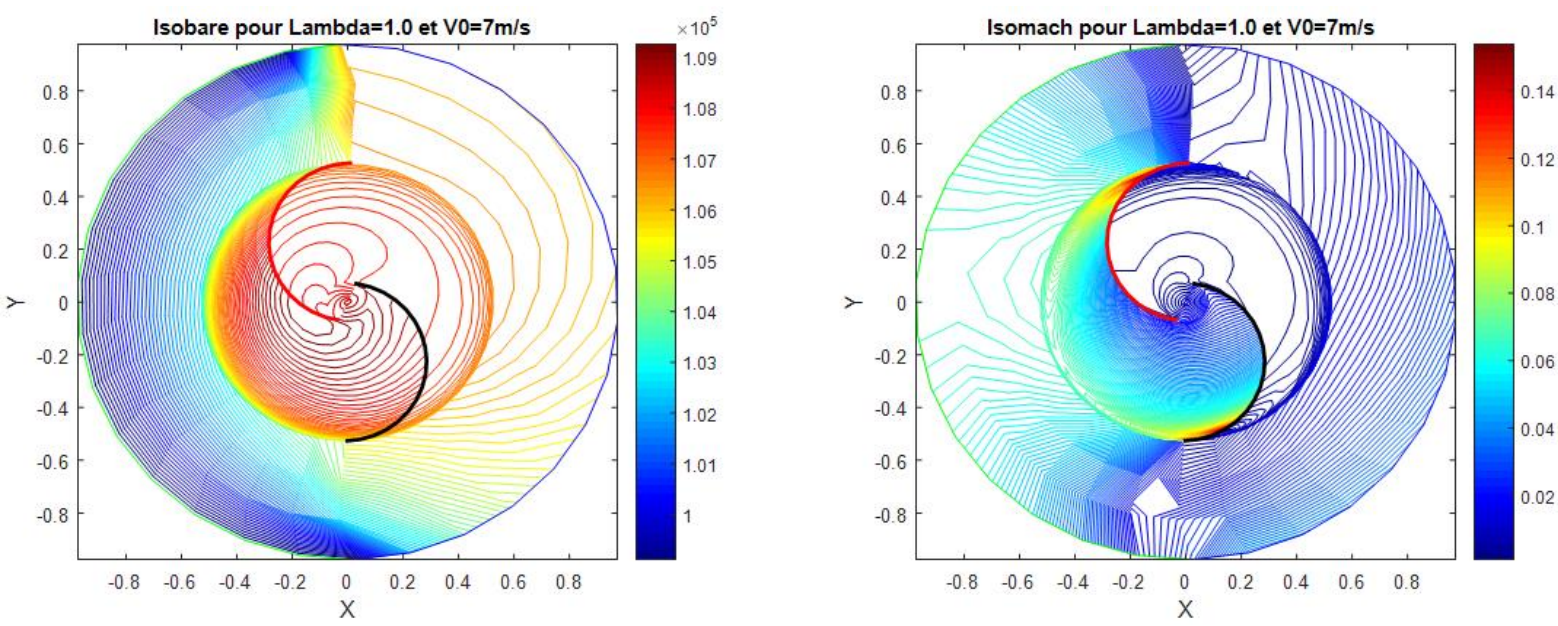

Figure 17. Courbes d'isobare à l'instant $t=11.83 \mathrm{~s}, \alpha=90^{\circ}$ après avoir effectué treize tours
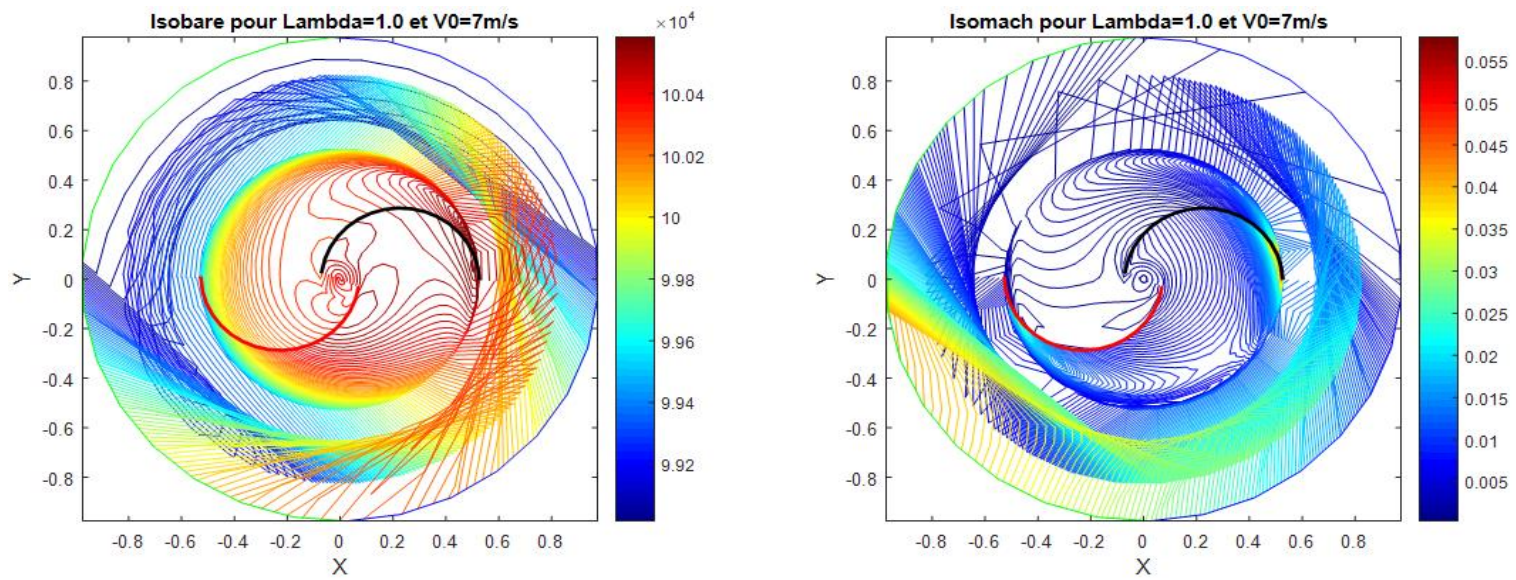

Figure 18. Courbes d'isobare à l'instant $t=11.94 \mathrm{~s}, \alpha=180^{\circ}$ après avoir effectué treize tours de plus 

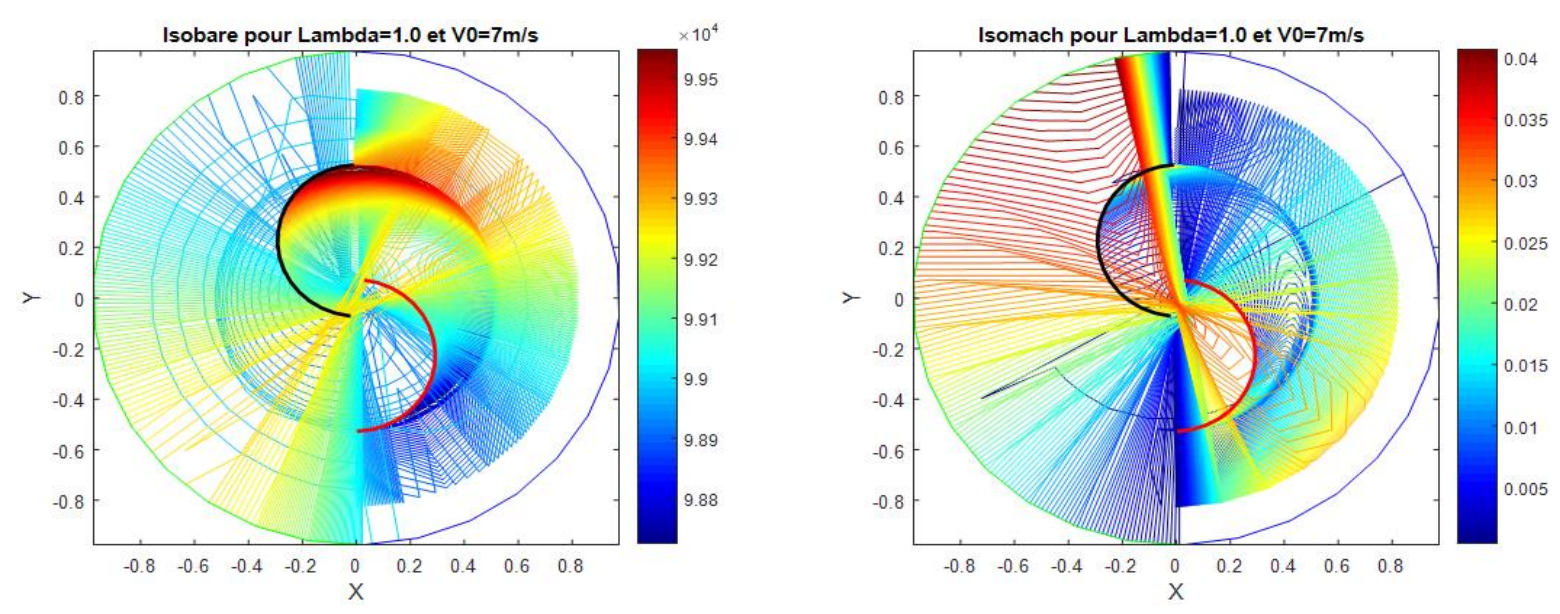

Figure 19. Courbes d'isobare à l'instant $t=12.06 s, \alpha=270^{\circ}$ après avoir effectué treize tours de plus
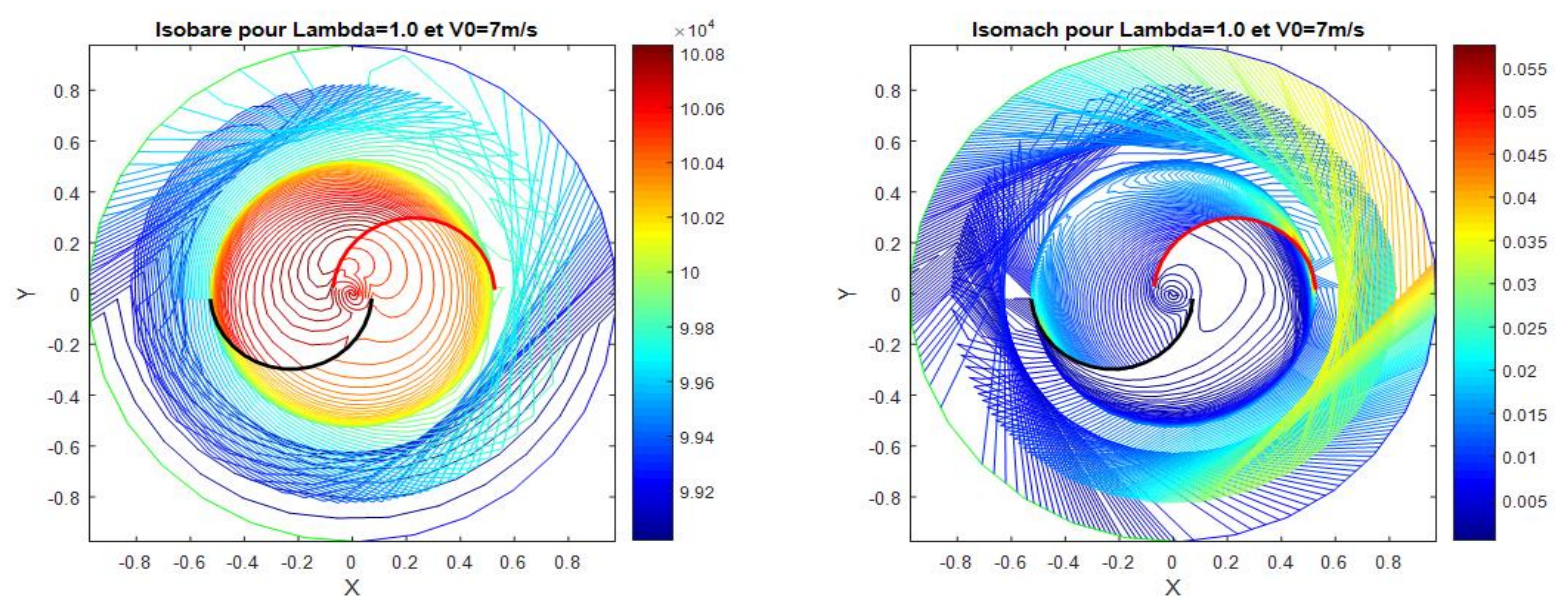

Figure 20. Courbes d'isobare à l'instant $t=12.18 \mathrm{~s}, \alpha=360^{\circ}$ après avoir effectué treize tours de plus
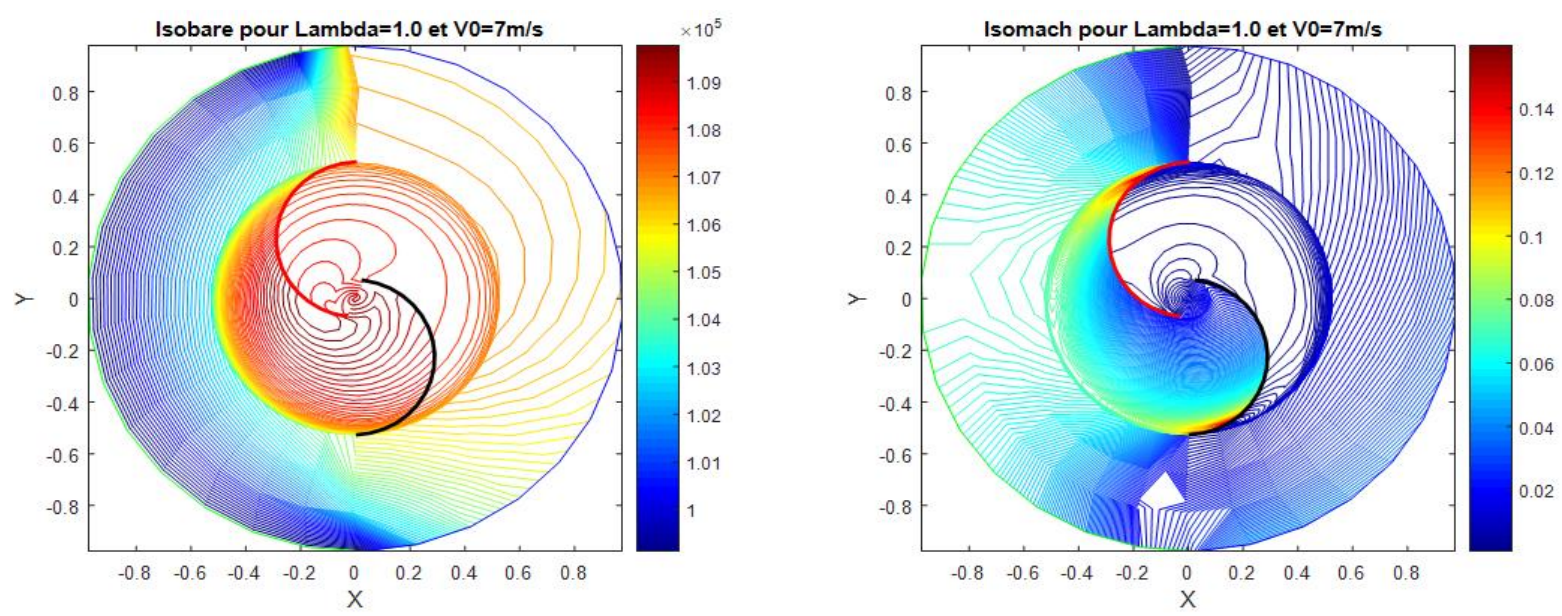

Figure 21. Courbes d'isobare à l'instant $t=12.29 \mathrm{~s}, \alpha=90^{\circ}$ après avoir effectué quatorze tours

Les figures 6-12 représentent les courbes isobare et iso-Mach pour différentes valeurs du paramètre d'avance $\lambda$, avec une vitesse absolue $\mathrm{V}=5 \mathrm{~m} / \mathrm{s}$, un nombre d'itérations IT $=43000$ et pendant une période $\mathrm{t}=0,23 \mathrm{~s}$. Nous avons remarqué que le paramètre d'avance a beaucoup d'influence sur la vitesse de rotation d'une éolienne. Sa valeur optimale est environ 0,9, mais cela dépend en partie des données géométriques d'une éolienne. Les figures 13 à 16 montrent que le débit des fluides est très 
varié ou perturbé au démarrage. La vitesse de l'écoulement de fluide est presque transsonique sur le recouvrement interne d'un rotor selon la figure 13 provoquant ainsi un tourbillon.

Les figures 13-21 montrent les mêmes résultats que précédemment, mais le paramètre d'avance et la vitesse absolue sont tous fixes, tandis que le nombre d'itérations varie. Les résultats de la simulation nous montrent que les phénomènes physiques rencontrés varient à chaque instant grâce à l'écoulement de fluide qui est instationnaire. Nous avons constaté que la zone la plus perturbée se situe au niveau des bords d'attaque, bords de fuite et surtout sur le recouvrement interne du rotor. Dans une zone correspondant à l'intrados de la pale en avance (figure 17-20), nous avons remarqué une dépression et une surpression sur l'intrados de la pale en retour (pale en noir). Cette différence de pression crée un couple qui fait tourner l'éolienne. La turbulence de ces deux zones est légèrement influencée par l'accélération des particules générée par la courbure des aubes et l'effet de rotation de la turbine. Nous observons également que les courbes isobares sont en corrélation avec les courbes d'iso-Mach relatives. C'est-à-dire dans la partie haute pression, la vitesse d'écoulement des fluides est faible. Ensuite, la loi de Bernoulli est vérifiée, car la pression varie inversement proportionnellement à la vitesse. Dans une étude instationnaire, la stabilité d'un système est atteinte si les résultats obtenus entre deux intervalles de temps successifs sont identiques. Cela est prouvé par les figures 16, 17 et $21:$ les résultats obtenus à l'instant $\mathrm{t}=11.83 \mathrm{~s}$ (13 tours de rotations d'un rotor) sont identiques aux résultats obtenus à l'instant $\mathrm{t}=12.29 \mathrm{~s}$ (14 tours d'un rotor).

A partir de six tours d'un rotor d'une éolienne, les résultats obtenus sont similaires. Pour obtenir un bon résultat, nous avons besoin de 12,29 secondes en temps réel, mais d'une durée de quatre jours en temps machine. Cette durée varie en fonction de la capacité des ordinateurs ainsi que des conditions aux limites fixées sur les frontières du domaine de calcul.

\section{Conclusion}

Cette étude a pour objectif d'analyser le comportement aérodynamique des fluides à l'intérieur d'un rotor d'une éolienne Savonius afin de l'améliorer. Ce type de fluide est modélisé par les équations d'Euler dont la résolution analytique est encore impossible que pour des structures simples et à faible degré de liberté [KAY 18]. Par conséquent, une résolution numérique par la méthode des différences finies explicites a été adoptée en utilisant un schéma de viscosité artificielle corrigé afin de minimiser le temps de calcul, mais sans fausser les résultats.

Les résultats de la simulation ont montré que l'écoulement des fluides autour d'un profil en mouvement est perturbé en raison des conditions initiales et des limites. Cependant, ces perturbations sont amorties pendant le calcul en raison de la viscosité numérique appliquée. Cette simulation dans un canal inter aubes d'une éolienne conventionnelle Savonius montre une dépression en aval et une surpression en amont. Cette variation de pression entre l'entrée et la sortie du domaine de calcul est inversement proportionnelle à celle de la vitesse. Par conséquent, l'éolienne est un abaisseur de pression ; c'est pourquoi on l'appelle turbine. Les résultats de notre code de calcul sont très fiables et efficaces. Toutefois, une démarche inverse sera appréciée afin d'améliorer la géométrie d'une machine pour obtenir à la puissance désirée.

Remerciements : Les auteurs souhaitent remercier toutes les personnes qui m'ont soutenu dans cette recherche. Nous pensons en particulier à l'équipe du Laboratoire MFSEA (Mécanique des Fluides et Systèmes Énergétiques Appliqués) de l'Université d'Antsiranana (Madagascar) avoir apporté leur expertise scientifique. 


\section{Bibliographie}

[BUT 13] BUTAUD P., AURELIEN B., THIBAUD M. et MICHEL M, Etude aérodynamique instationnaire d'un rotor éolien de Savonius : mise en évidence de l'influence de la portance. Congrès Mécanique $(21 ; 2013$; Bordeaux), Aug 2013, France. <hal-00904222>.

[DEB 13] DEBNATH P. et RAJAT G., Flow Physics analysis of Three-bucket Helical Savonius rotor at 90-degree twist angle using CFD", International Journal of Modern Engineering Research (IJMER) Vol.3, Issue.2, March-April. 2013 pp-739-746, ISSN: 2249-664.

[DOB 12] DOBREV I. et FAWAZ M., Exploring the Flow around a Savonius Wind Turbine, 16th Int Symposium on Applications of Laser Techniques to Fluid Mechanics, Lisbon, Portugal, 09-12 July, 2012.

[DWI 16] DWIYANTORO B. A., TRIYOGI Y. et VIVIEN S., Structural design optimization of vertical axis wind turbine type Darrieus-Savonius, ARPN Journal of Engineering and Applied Sciences, Vol. 11, No. 2, Janvier 2016.

[ELK 15] EL KHCHINE Y., SRITI M. et OUARIAGLI M, «Simulation des performances aérodynamiques d'une éolienne à axe horizontal », 12ème Congrès de Mécanique 21-24 Avril 2015. Casablanca, Maroc.

[GHA 14] GHANEGAONKAR P. M., KAWADE R. K. et GARG S., Conceptual Model of Vertical Axis Wind Turbine and CFD analysis, International Journal of Innovative Research in Advanced Engineering, (IJIRAE), p. 76-81, Volume 1, Issue 3, May 2014.

[HAD 13] HADI ALI M., Experimental Comparison Study for Savonius Wind Turbine of Two \& Three Blades at Low Wind Speed, International Journal of Modern Engineering Research (IJMER), Vol. 3, Issue. 5, Sep - Oct. 2013 pp2978-2986. ISSN: 2249-6645.

[KAN 13] KANG C., XIN Y. et YULI W., Turbulent Flow Characteristics and Dynamics Response of a Vertical-Axis Spiral Rotor, Energies 2013, 6(6), 2741-2758; https://doi.org/10.3390/en6062741.

[KAY 18] KANYIKI T., Simulation par la Méthode des Eléments Finis du Comportement Vibratoire d'un Rotor, Université de Lubumbashi, République Démocratique du Congo, ISTE Ltd. London, UK - openscience.fr, 2018. DOI : 10.21494/ISTE.OP.2018.0305.

[MAR 17] MARCHEVSKY ILIA K. et VALERIA V. P., Numerical Simulation of Wind Turbine Rotors Autorotation by Using the Modified LS-STAG Immersed Boundary Method, October 2017, International Journal of Rotating Machinery 2017:1-7, https://doi.org/10.1155/2017/6418108.

[NOU 15] NOUIOUA A et RABAH D. A., Modélisation d'un écoulement pulsatif et oscillatoire autour d'une pale d'éolienne HAWT Application au décrochage dynamique, Université des Sciences et de la Technologie Houari Boumediene, Alger, Algérie. Revue des Energies Renouvelables, Vol. 18, №. 1, Mars 2015.

[PRU 18] PRUD'HOMME R., Modélisation d'interfaces avec phase fluide, Sorbonne Université, CNRS, Institut Jean Le Rond d'Alembert, Paris, ISTE Ltd. London, UK - openscience.fr, 2018. DOI : 10.21494/ISTE.OP.2018.0214.

[SON16] SONG L., HONG-ZHAO L. et ZONG-XIAO Y, Orthogonal Analysis Based Performance Optimization for Vertical Axis Wind Turbine, Mathematical Problems in Engineering, Volume 2016, Article ID 6241360, http://dx.doi.org/10.1155/2016/6241360.

[SOS 18] SOSSEY-ALAOUI. I. et RADI B., Une étude aérodynamique d'une pale d'éolienne à axe horizontal, Laboratoire LIMII, Université Hassan, Maroc, ISTE OpenScience, Londres, 2018 ; https://www.openscience.fr/IMG/pdf/iste incertfia18v2n1 4.pdf.

[WAH 13] WAHYUDI B, SUDJITO S., SLAMET W., et Widhiyanuriyawan D., A Simulation Study of Flow and Pressure Distribution Patterns in and around of Tandem Blade Rotor of Savonius (TBS) Hydrokinetic Turbine Model, Journal of Clean Energy Technologies, Vol. 1, No. 4, October 2013. DOI:10.7763/JOCET. 2013.V1.65.

[WID 12] WIDODO W. S., CHIN A. C., HAERYIP S., et YUHAZRI M. Y., Design and analysis of 5 kw Savonius rotor blade, Global Engineers \& Technologists Review, Vol.2 No.8 (2012).

[ZHA 17] ZHANG B., BAOWEI S., ZHAOYONG M., WENLONG T., BOYANG et BO L., A Novel Parametric Modeling Method and Optimal Design for Savonius Wind Turbines, www.mdpi.com/journal/energies, March 2017. Energies 2017, 10, 301; doi:10.3390/en10030301. 